PROCEEDINGS OF THE

AMERICAN MATHEMATICAL SOCIETY

Volume 138, Number 8, August 2010, Pages 2923-2937

S 0002-9939(10)10308-6

Article electronically published on April 14, 2010

\title{
NUMBER OF LEAST AREA PLANES IN GROMOV HYPERBOLIC 3-SPACES
}

\author{
BARIS COSKUNUZER
}

(Communicated by Alexander N. Dranishnikov)

\begin{abstract}
We show that for a generic simple closed curve $\Gamma$ in the asymptotic boundary of a Gromov hyperbolic 3-space with cocompact metric $X$, there exists a unique least area plane $\Sigma$ in $X$ such that $\partial_{\infty} \Sigma=\Gamma$. This result has interesting topological applications for constructions of canonical 2-dimensional objects in Gromov hyperbolic 3-manifolds.
\end{abstract}

\section{INTRODUCTION}

Let $X$ be a Gromov hyperbolic 3-space with cocompact metric, and let $S_{\infty}^{2}(X)$ be the sphere at infinity of $X$. Let $\Gamma$ be a given simple closed curve in $S_{\infty}^{2}(X)$. The asymptotic Plateau problem asks the existence of a least area plane $\Sigma$ in $X$ with asymptotic boundary $\Gamma$, i.e. $\partial_{\infty} \Sigma=\Gamma$. The author gave a positive answer to this question and showed the existence of such least area planes asymptotic to a given curve in $S_{\infty}^{2}(X)$ in $\mathrm{Co1}$.

For the analogous problem for $\mathbf{H}^{3}$, Michael Anderson showed the existence of a least area plane $\Sigma \subset \mathbf{H}^{3}$ asymptotic to a given simple closed curve $\Gamma \subset S_{\infty}^{2}\left(\mathbf{H}^{3}\right)$ in [A1, A2. Later, by using topological techniques instead of geometric measure theory, Gabai obtained a similar result for a hyperbolic 3-space with cocompact metric in Ga.

On the other hand, on the number of the least area planes for a given simple closed curve in the asymptotic sphere, there are a few results so far. In A1, Anderson showed that if the given asymptotic boundary $\Gamma \subset S_{\infty}^{2}\left(\mathbf{H}^{3}\right)$ bounds a convex domain in $S_{\infty}^{2}\left(\mathbf{H}^{3}\right)$, then there exists a unique least area plane in $\mathbf{H}^{3}$. Then, Hardt and Lin generalized this result to the simple closed curves bounding star shaped domains in $S_{\infty}^{2}\left(\mathbf{H}^{3}\right)$ in [HL. Recently, the author showed that a generic simple closed curve in $S_{\infty}^{2}\left(\mathbf{H}^{3}\right)$ bounds a unique least area plane in $\mathbf{H}^{3}$ in [Co2, Co4.

For the number of least area planes in a Gromov hyperbolic space spanning a given curve in the asymptotic boundary, there is no result so far. In this paper, we will prove that a generic simple closed curve in $S_{\infty}^{2}(X)$ bounds a unique least area plane in $X$. Our main result is as follows:

Received by the editors October 15, 2009 and, in revised form, December 2, 2009.

2010 Mathematics Subject Classification. Primary 53A10, 57M50.

The author is partially supported by EU-FP7 Grant IRG-226062, TUBITAK Grant 107T642 and a TUBA-GEBIP Award. 
Theorem 3.3. Let $X$ be a Gromov hyperbolic 3-space with cocompact metric. Let $A$ be the space of simple closed curves in $S_{\infty}^{2}(X)$ and $A^{\prime} \subset A$ be the subspace consisting of the simple closed curves in $S_{\infty}^{2}(X)$ bounding a unique least area plane in $X$. Then, $A^{\prime}$ is generic in $A$; i.e. $A-A^{\prime}$ is a set of first category.

This is the first result in this direction for Gromov hyperbolic spaces. The techniques are very general, and they can be applied to many similar settings. Note also that the unique planes obtained in this theorem are also properly embedded (See Remark 3.1.) Furthermore, there are interesting applications of this result. Namely, when $\Gamma_{1}$ or $\Gamma_{2}$ is generic, the following conjecture is true.

Disjoint Planes Conjecture. Let $\Gamma_{1}, \Gamma_{2}$ be simple closed curves in $S_{\infty}^{2}(X)$. If $\Gamma_{1}$ and $\Gamma_{2}$ do not cross each other (i.e. they are the boundaries of disjoint open regions in $S_{\infty}^{2}(X)$ ), then any distinct least area planes $\Sigma_{1}, \Sigma_{2}$ in $X$ with asymptotic boundary $\Gamma_{1}, \Gamma_{2}$ are disjoint.

If this conjecture is true in general, it can be shown that for a genuine lamination $\Lambda$ in a closed atorodial 3-manifold $M$, by inducing a $\pi_{1}$-invariant family of curves $\partial_{\infty} \widetilde{\Lambda}$ in $S_{\infty}^{2}(\widetilde{M})$ with continuous extension property, $\Lambda$ can be modified to $\Delta$, which is a genuine lamination with least area leaves. This can be done by replacing the leaves of $\widetilde{\Lambda}$ with least area planes $\widetilde{\Delta}$, we get a genuine lamination $\Delta$ in $M$ with least area leaves. By the Disjoint Planes Conjecture, $\widetilde{\Delta}$ projects down to an embedded genuine lamination $\Delta$ in $M$. Hence, if the induced curves in $S_{\infty}^{2}(\widetilde{M})$ are generic, with Theorem 3.3, it is possible to modify a given genuine $\Lambda$ to the one with least area leaves $\Delta$ in $M$. Of course, it is possible to do the same for any essential 2-dimensional object in a Gromov hyperbolic 3-manifold.

The organization of the paper is as follows: In the next section we will cover some basic results which will be used in the following sections. In section 3, we will prove the main result of the paper. Then in section 4, we will show some applications of the main result and give an example which shows a subtle point of the Disjoint Planes Conjecture. Finally in section 5, we will have some final remarks.

\section{Preliminaries}

A 3-manifold $M$ is called a Gromov hyperbolic manifold if its fundamental group $\pi_{1}(M)$ is a word hyperbolic (or Gromov hyperbolic) group $\mathrm{Gr}$. We call $X$ a Gromov hyperbolic 3-space with cocompact metric if $X$ is the universal cover of a Riemannian closed orientable irreducible Gromov hyperbolic 3-manifold $M$, where the metric on $X$ is induced by $M$. By Bestvina-Mess [BM], $X$ is homeomorphic to an open ball in $\mathbf{R}^{3}$, and it has a natural compactification $\bar{X}$, where $\bar{X}=X \cup \partial_{\infty} X$. They also show that $\partial_{\infty} X$ is homeomeorphic to a sphere, called the sphere at infinity $S_{\infty}^{2}(X)$, and a point on $S_{\infty}^{2}(X)$ corresponds to an equivalence class of infinite rays in $X$, where two rays are equivalent if they are within finite Hausdorff distance from each other. If $A$ is a subset of $X$, then the asymptotic boundary $\partial_{\infty} A$ of $A$ is defined as $\partial_{\infty} A=\bar{A} \cap S_{\infty}^{2}(X)$, where $\bar{A}$ is the closure of $A$ in $\bar{X}$.

We call a disk $D$ a least area disk if $D$ has the smallest area among the disks with the same boundary $\partial D$. A plane is a subset of $X$ with the topological type of a disk and is complete with respect to the induced path metric from $X$. We call a plane $P$ a least area plane if any subdisk in the plane $P$ is a least area disk.

A codimension-1 lamination $\sigma$ in $X$ is a foliation of a closed subset of $X$ with 2-manifolds (leaves) such that $X$ is covered by charts of the form $I^{2} \times I$, where a 
leaf passes through a chart in a slice of the form $I^{2} \times\{p\}$ for $p \in I$. Here and later, we abuse notation by letting $\sigma$ also denote the underlying space of its lamination.

Definition 2.1. The sequence $\left\{D_{i}\right\}$ of smooth embedded disks in a Riemannian manifold $X$ converges to the lamination $\sigma$ if

- $\sigma=\left\{x=\lim x_{i} \mid x_{i} \in D_{i},\left\{x_{i}\right\}\right.$ is a convergent sequence in $\left.X\right\}$.

- $\sigma=\left\{x=\lim x_{n_{i}} \mid x_{i} \in D_{i},\left\{x_{i}\right\}\right.$ has a convergent subsequence $\left\{x_{n_{i}}\right\}$ in $\left.X\right\}$.

- For any $x \in \sigma$, there exists a sequence $\left\{x_{i}\right\}$ with $x_{i} \in D_{i}$ and $\lim x_{i}=x$ such that there exist embeddings $f_{i}: D^{2} \rightarrow D_{i}$ which converge in the $C^{\infty}$ topology to a smooth embedding $f: D^{2} \rightarrow L_{x}$, where $x_{i} \in f_{i}\left(\operatorname{Int}\left(D^{2}\right)\right)$, and $L_{x}$ is the leaf of $\sigma$ through $x$, and $x \in f\left(\operatorname{Int}\left(D^{2}\right)\right)$.

We call such a lamination $\sigma$ a $D^{2}$-limit lamination $\mathrm{Ga}$.

In other words, the sequence $\left\{D_{i}\right\}$ is such that the set of the limits of all $\left\{x_{i}\right\}$ with $x_{i} \in X$ and the set of the limits of the subsequences are the same (the first condition in the definition). Note that this is an essential condition on $\sigma$ to be a collection of pairwise disjoint embedded disks. Otherwise, one might simply take a sequence such that $D_{2 i+1} \subset \Sigma_{1}$ and $D_{2 i} \subset \Sigma_{2}$, where $\Sigma_{1}$ and $\Sigma_{2}$ are intersecting least area planes and $\left\{D_{2 i+1}\right\}$ exhaust $\Sigma_{1}$ and $\left\{D_{2 i}\right\}$ exhaust $\Sigma_{2}$. Then, without the first condition ( $\sigma$ being just the union of limit points), $\sigma=\Sigma_{1} \cup \Sigma_{2}$ in this case, which is not a collection of pairwise disjoint embedded disks. However, the first condition forces $\sigma$ to be either $\Sigma_{1}$ or $\Sigma_{2}$, not the union of them. By similar reasons, we will see later that this condition is also important to ensure the embeddedness of the disks in the collection $\sigma$.

Theorem 2.1 ([Co1]). Let $\Gamma$ be a simple closed curve in $S_{\infty}^{2}(X)$ where $X$ is a Gromov hyperbolic 3-space with cocompact metric. Then there exists a $D^{2}$-limit lamination $\sigma \subset X$ by least area planes $\left\{\Sigma_{i}\right\}$ with $\partial_{\infty} \Sigma_{i}=\Gamma$.

Remark 2.1. Gabai proved this theorem for hyperbolic 3-spaces with cocompact metric in Ga.

Note that the basic background material and relevant results on the asymptotic Plateau problem can be found in the survey article [Co6]. The following lemma is often called the Meeks-Yau exchange roundoff trick.

Lemma 2.2 ( $\mathrm{MY}]$ ). If $D_{1}$ and $D_{2}$ are embedded least area disks in a manifold $M$ such that $D_{1} \cap \partial D_{2}=\emptyset$ and $D_{2} \cap \partial D_{1}=\emptyset$, then $D_{1} \cap D_{2}=\emptyset$.

The following lemma is known as the maximum principle for minimal surfaces. It describes the intersection of two minimal surfaces near a point of tangency as a monkey saddle [HS].

Lemma 2.3. Let $M$ be a Riemannian manifold and $S_{1}$ and $S_{2}$ be minimal surfaces in $M$. Assume that $S_{1}$ and $S_{2}$ are tangent to each other at $x$ in $M$. Then either $S_{1}$ and $S_{2}$ coincide or there is a neighborhood of $x$ in $M$ which is parameterized by a $C^{1}$ coordinate chart $\left(x_{1}, x_{2}, x_{3}\right)$ such that $S_{1}$ is given by $x_{3}=0$ and $S_{2}$ is given by $x_{3}=\operatorname{Re}\left(x_{1}+i x_{2}\right)^{n}$ for some $n \geq 2$ (a monkey saddle).

In particular, this lemma says that one minimal surface cannot have a tangential intersection with another minimal surface by lying in one side of the other (maximum principle). 


\section{Generic uniqueness of Least area Planes}

In this section, we will prove that the space of simple closed curves in $S_{\infty}^{2}(X)$ bounding a unique least area plane in $X$ is generic in the space of simple closed curves in $S_{\infty}^{2}(X)$.

The short outline of the technique is the following: Let $\Gamma_{0}$ be a simple closed curve in $S_{\infty}^{2}(X)$. First, we will show that either there exists a unique least area plane $\Sigma_{0}$ in $X$ with $\partial_{\infty} \Sigma_{0}=\Gamma_{0}$ or there exist two disjoint least area planes $\Sigma_{0}^{+}, \Sigma_{0}^{-}$ in $X$ with $\partial_{\infty} \Sigma_{0}^{ \pm}=\Gamma_{0}$.

Now, take a small neighborhood $N\left(\Gamma_{0}\right) \subset S_{\infty}^{2}(X)$ which is an annulus. Then foliate $N\left(\Gamma_{0}\right)$ by simple closed curves $\left\{\Gamma_{t}\right\}$ where $t \in(-\epsilon, \epsilon)$, i.e. $N\left(\Gamma_{0}\right) \simeq \Gamma \times$ $(-\epsilon, \epsilon)$. By the above fact, for any $\Gamma_{t}$ either there exists a unique least area plane $\Sigma_{t}$ or there are two canonical least area planes $\Sigma_{t}^{ \pm}$disjoint from each other. Also, since these are least area planes, if they have disjoint asymptotic boundary, then they are disjoint by the Meeks-Yau exchange roundoff trick. This means that if $t_{1}<t_{2}$, then $\Sigma_{t_{1}}$ is disjoint and below $\Sigma_{t_{2}}$ in $X$. Consider this collection of least area planes. Note that for curves $\Gamma_{t}$ bounding more than one least area plane, we have a canonical region $N_{t}$ in $X$ between the disjoint least area planes $\Sigma_{t}^{ \pm}$.

Now, $N(\Gamma)$ separates $S_{\infty}^{2}(X)$ into two parts. Take a properly embedded line $\beta \subset X$ which is asymptotic to two points belonging to these two different parts. Also, let $\beta$ be transverse to the collection of these least area planes asymptotic to the curves in $\left\{\Gamma_{t}\right\}$. Only a finite segment $\hat{\beta}$ of $\beta$ intersects the entire collection. Let the length of $\hat{\beta}$ be $C$.

Now, the idea is to consider the thickness of the neighborhoods $N_{t}$ assigned to the asymptotic curves $\left\{\Gamma_{t}\right\}$. Let $s_{t}$ be the total length of the segment (or segments) $I_{t}$ of $\hat{\beta}$ between $\Sigma_{t}^{+}$and $\Sigma_{t}^{-}$, which is the width of $N_{t}$ assigned to $\Gamma_{t}$. Then, the curves $\Gamma_{t}$ bounding more than one least area plane have positive width and contribute to total thickness of the collection, and the curves bounding unique least area plane have 0 width and do not contribute to the total thickness. Since $\sum_{t \in(-\epsilon, \epsilon)} s_{t}<C=|\hat{\beta}|$, the total thickness is finite. This implies for only countably many $t \in(-\epsilon, \epsilon), s_{t}>0$, i.e. $\Gamma_{t}$ bounds more than one least area plane. For the remaining uncountably many $t \in(-\epsilon, \epsilon), s_{t}=0$, and there exists a unique least area plane for those $t$. This proves that the space of Jordan curves bounding a unique plane is dense in the space of Jordan curves in $S_{\infty}^{2}(X)$. Then, by using similar arguments, we will show this space is not only dense, but also generic.

First, we will show that if two least area planes have disjoint asymptotic boundaries, then they are disjoint.

Lemma 3.1. Let $\Gamma_{1}$ and $\Gamma_{2}$ be two disjoint simple closed curves in $S_{\infty}^{2}(X)$. If $\Sigma_{1}$ and $\Sigma_{2}$ are least area planes in $X$ with $\partial_{\infty} \Sigma_{i}=\Gamma_{i}$, then $\Sigma_{1}$ and $\Sigma_{2}$ are disjoint, too.

Proof. Assume that $\Sigma_{1} \cap \Sigma_{2} \neq \emptyset$. Since $\partial_{\infty} \Sigma_{i}=\Gamma_{i}$ and $\Gamma_{1}, \Gamma_{2}$ are two disjoint simple closed curves in $S_{\infty}^{2}(X)$, the intersection $\Sigma_{1} \cap \Sigma_{2}$ must belong to a compact subset $K$ of $X$. Also, as $\Sigma_{1}$ and $\Sigma_{2}$ are complete minimal surfaces, the intersection between $\Sigma_{1}$ and $\Sigma_{2}$ consists of simple closed curves $\left\{\gamma_{i}\right\}$ or infinite lines $\left\{l_{j}\right\}$. Note that these $\left\{\gamma_{i}\right\}$ and $\left\{l_{j}\right\}$ might only intersect each other in isolated points by Lemma 2.3; i.e. the set $\left(\gamma_{i} \cap \gamma_{j}\right) \cup\left(\gamma_{i} \cap l_{j}\right) \cup\left(l_{i} \cap l_{j}\right)$ consists of isolated points in $X$. 
First, we show that there is no simple closed curve in the intersection $\Sigma_{1} \cap \Sigma_{2}$. Assume that the intersection contains a simple closed curve $\gamma$. Since $\Sigma_{1}$ and $\Sigma_{2}$ are both minimal, the intersection must be transverse on a subarc of $\gamma$ by maximum principle. Now, $\gamma$ bounds two least area disks $D_{1}$ and $D_{2}$ in $X$, with $D_{i} \subset \Sigma_{i}$. Now, take a larger subdisk $E_{1}$ of $\Sigma_{1}$ containing $D_{1}$, i.e. $D_{1} \subset E_{1} \subset \Sigma_{1}$. By definition, $E_{1}$ is also a least area disk. Now, modify $E_{1}$ by swapping the disks $D_{1}$ and $D_{2}$. Then, we get a new disk $E_{1}^{\prime}=\left\{E_{1}-D_{1}\right\} \cup D_{2}$. Now, $E_{1}$ and $E_{1}^{\prime}$ have the same area, but $E_{1}^{\prime}$ have a folding curve along $\gamma$. By smoothing out this curve as in $\mathrm{MY}$, we get a disk with smaller area, which contradicts $E_{1}$ being the least area (this is the Meeks-Yau exchange roundoff trick).

Now, we show that there is no infinite line in the intersection $\Sigma_{1} \cap \Sigma_{2}$. Assume that $l$ is an infinite line in this intersection. Let $\mathcal{A}$ be the annulus in $S_{\infty}^{2}(X)$ with $\partial \mathcal{A}=\Gamma_{1} \cup \Gamma_{2}$. Then, let $\widehat{\Gamma}$ be an essential curve in $\mathcal{A}$ separating $\Gamma_{1}$ and $\Gamma_{2}$ in $S_{\infty}^{2}(X)$. Let $\widehat{\Sigma}$ be a properly embedded least area plane in $S_{\infty}^{2}(X)$ with $\partial_{\infty} \widehat{\Sigma}=\widehat{\Gamma}$ given by Co3. By their construction, $\widehat{\Sigma}$ is separating in $X$ such that $X-\widehat{\Sigma}=X^{+} \cup X^{-}$and $\partial_{\infty} X^{ \pm}=\Omega^{ \pm}$, where $S_{\infty}^{2}(X)-\widehat{\Gamma}=\Omega^{+} \cup \Omega^{-}$. Say $\Gamma_{1} \subset \Omega^{+}$and $\Gamma_{2} \subset \Omega^{-}$. Hence, if $\Sigma_{1} \cap \Sigma_{2} \neq \emptyset$, then $\Sigma_{1} \cap \widehat{\Sigma} \neq \emptyset$ or $\Sigma_{2} \cap \widehat{\Sigma} \neq \emptyset$. Otherwise, $\Sigma_{1} \subset X^{+}$and $\Sigma_{2} \subset X^{-}$, hence they would be disjoint. So, let $\Sigma_{1} \cap \widehat{\Sigma} \neq \emptyset$. The intersection cannot contain a simple closed curve as shown in the previous paragraph. Hence, there is an infinite line $l^{\prime}$ in a compact set $K^{\prime}$ and in the intersection $\Sigma_{1} \cap \widehat{\Sigma}$. However, $\widehat{\Sigma}$ is properly embedded, so $\widehat{\Sigma}$ must contain the limit point of $l^{\prime}$. However, this contradicts the characterization of intersection of minimal surfaces (maximum principle) by Lemma 2.3. So, there is no infinite line in the intersection $\Sigma_{1} \cap \Sigma_{2}$. The proof follows.

The following lemma is essential for our technique. Mainly, the lemma says that for any given simple closed curve $\Gamma$ in $S_{\infty}^{2}(X)$, either there exists a unique least area plane $\Sigma$ in $X$ asymptotic to $\Gamma$ or there exist two canonical least area planes $\Sigma^{ \pm}$in $X$ which are asymptotic to $\Gamma$ and disjoint from each other.

Lemma 3.2. Let $X$ be a Gromov hyperbolic 3-space with cocompact metric, and let $\Gamma$ be a simple closed curve in $S_{\infty}^{2}(X)$. Then either there exists a unique least area plane $\Sigma$ in $X$ with $\partial_{\infty} \Sigma=\Gamma$ or there are two canonical disjoint extremal least area planes $\Sigma^{+}$and $\Sigma^{-}$in $X$ with $\partial_{\infty} \Sigma^{ \pm}=\Gamma$. Moreover, any least area plane $\Sigma^{\prime}$ with $\partial_{\infty} \Sigma^{\prime}=\Gamma$ is disjoint from $\Sigma^{ \pm}$, and it is captured in the region bounded by $\Sigma^{+}$ and $\Sigma^{-}$in $X$.

Proof. Let $\Gamma$ be a simple closed curve in $S_{\infty}^{2}(X)$. $\Gamma$ separates $S_{\infty}^{2}(X)$ into two parts, say $\Omega^{+}$and $\Omega^{-}$, which are open disks. Define sequences of pairwise disjoint simple closed curves $\left\{\Gamma_{i}^{+}\right\}$such that $\Gamma_{i}^{+}=\partial E_{i}$, where $E_{i}$ is a closed disk in $\Omega^{+}$for any $i$ and $E_{i} \subset \operatorname{Int}\left(E_{j}\right)$ for any $i<j$. Moreover, $\Omega^{+}=\bigcup_{i} E_{i}$ and $\Gamma_{i}^{+} \rightarrow \Gamma$.

By Theorem 2.1, for any $\Gamma_{i}^{+} \subset S_{\infty}^{2}(X)$ there exists a least area plane $\Sigma_{i}^{+}$with $\partial_{\infty} \Sigma_{i}^{+}=\Gamma_{i}^{+}$. Note that these least area planes are separating in $X$ by their construction in Theorem 2.1. Also, let $p^{+} \in \operatorname{Int}\left(E_{1}\right) \subset \Omega^{+}$and $p^{-} \in \Omega^{-}$be two points belonging different components of $S_{\infty}^{2}\left(\mathbf{H}^{3}\right)-\Gamma$. Let $\beta$ be a proper line in $X$ asymptotic to $p^{+}$and $p^{-}$. Since each $\overline{\Sigma_{i}^{+}}$is separating in $\bar{X}, \beta$ intersects $\Sigma_{i}^{+}$for any $i$. Let $x_{i}$ be a point in $\Sigma_{i}^{+} \cap \beta$ for any $i$.

Now, we define the sequence of least area disks. Let $D_{i}$ be a least area disk in $\Sigma_{i}^{+}$ containing $x_{i}$ such that $i<d_{X}\left(x_{i}, \partial D_{i}\right)<i+1$, where $d_{X}$ is the extrinsic distance in $X$. We claim that the sequence of least area disks $\left\{D_{i}\right\}$ in $X$ converge to a 
nonempty lamination $\sigma$. As $\alpha_{i}=\partial D_{i}$ converges to $\Gamma$, by Theorem $2.1, D_{i}$ converges to a lamination $\sigma$ (possibly empty) by least area planes with $\partial_{\infty} \sigma=\Gamma$. Now, we show that $\sigma$ is a nonempty lamination by least area planes. Since $p^{+} \in \Omega^{+}$and $p^{-} \in \Omega^{-}$where $\Omega^{ \pm}$are open disks in $S_{\infty}^{2}(X)$, we can find a sufficiently small $\epsilon$ such that $B_{\epsilon}\left(p^{+}\right) \subset \operatorname{Int}\left(E_{1}\right)$ and $B_{\epsilon}\left(p^{-}\right) \subset \Omega^{-}$. Let $\gamma^{+}=\partial B_{\epsilon}\left(p^{+}\right)$and $\gamma^{-}=\partial B_{\epsilon}\left(p^{-}\right)$. Then, $\gamma^{ \pm}$are two simple closed curves disjoint from $\Gamma$. By Theorem 2.1, there are least area planes $P^{+}$and $P^{-}$in $X$ with $\partial_{\infty} P^{ \pm}=\gamma^{ \pm}$. By Lemma 3.1, $P^{ \pm}$are disjoint from $\Sigma_{i}^{+}$for each $i$. So, the finite segment $\hat{\beta}$ of $\beta$ between $P^{+}$and $P^{-}$ contains all intersection points $\Sigma_{i}^{+} \cap \beta$ for any $i$. Hence, $\left\{x_{i}\right\} \subset \hat{\beta}$. Since $\hat{\beta}$ is a finite segment, it is compact, and the sequence $\left\{x_{i}\right\}$ has a limit point $x$. This shows that the sequence $\left\{D_{i}\right\}$ has a nonempty limit. So, $\sigma$ is a nonempty lamination by least area planes with $\partial_{\infty} \sigma=\Gamma$, as claimed.

Now, we want to show that $\sigma$ consists of only one least area plane $\mathcal{P}$, i.e. $\sigma=\mathcal{P}$. Assume that $\sigma$ contains more than one least area plane. Recall that $\sigma$ is a collection of disjoint least area planes asymptotic to $\Gamma$ and $\sigma$ is a closed subset of $X$. Note that each plane $\mathcal{P}$ in $\sigma$ is separating in $X$ by construction, by Theorem 4.3, Step 4 of Co1. (or Proposition 3.9, Step 3 in Ga ). Consider the components of $X-\sigma$. Let $X^{+}$be the component of $X-\sigma$ with $\partial_{\infty} X^{+}=\Omega^{+}$. Note that as $\sigma$ is closed, each component of $X-\sigma$ is open, and so is $X^{+}$. Since for each $i, D_{i} \subset \Sigma_{i}^{+}$and $\partial_{\infty} \Sigma_{i}^{+}=\Gamma_{i}^{+} \subset \Omega^{+}$, by Lemma 3.1, $D_{i} \subset X^{+}$. Since each plane $\mathcal{P}$ in $\sigma$ is separating, there is a plane $\mathcal{P}_{1}$ such that $\mathcal{P}_{1}=\partial X^{+}$. Let $\mathcal{P}_{2}$ be another least area plane in $\sigma$. We claim that there cannot be such a plane $\mathcal{P}_{2}$ because of the special properties of the sequence of least area disks $\left\{D_{i}\right\}$. Let $Y^{+}$be the component of $X-\mathcal{P}_{2}$ with $\partial_{\infty} Y^{+}=\Omega^{+}$. Clearly, $X^{+} \subset Y^{+}$. Moreover, since either there exists an open complementary region (a component of $X-\sigma$ ) or there is an open region foliated by least area planes in $\sigma$ between $\mathcal{P}_{1}$ and $\mathcal{P}_{2}, X^{+} \subsetneq \operatorname{Int}\left(Y^{+}\right)$. This means $\mathcal{P}_{1}$ forms a barrier between the sequence of least area disks $\left\{D_{i}\right\}$ and $\mathcal{P}_{2}$. In other words, the sequence $\left\{D_{i}\right\}$ cannot reach $\mathcal{P}_{2}$, so $\mathcal{P}_{2}$ cannot be in the limit. More precisely, if $q \in \mathcal{P}_{2}$, since $D_{i} \subset X^{+}$for any $i$, and $X^{+} \subsetneq \operatorname{Int}\left(Y^{+}\right)$, there is no sequence $\left\{q_{i}\right\}$ with $q_{i} \in D_{i}$ such that $q_{i} \rightarrow q$. So, $\mathcal{P}_{2}$ cannot be in the limit of $\left\{D_{i}\right\}$. Hence, this shows that $\sigma$ consists of only one plane $\mathcal{P}_{1}$, i.e. $\sigma=\mathcal{P}_{1}$. Call this least area plane $\mathcal{P}_{1}$ as $\Sigma^{+}$. Similarly by defining a similar sequence of simple closed curves converging to $\Gamma$ in $\Omega^{-}$, we can define the least area plane $\Sigma^{-}$.

Now, let $\Sigma^{\prime}$ be any other least area plane in $X$ with $\partial_{\infty} \Sigma^{\prime}=\Gamma$. If $\Sigma^{\prime} \cap \Sigma^{+} \neq \emptyset$, then some part of $\Sigma^{\prime}$ must be above $\Sigma^{+}$. Since $\Sigma^{+}=\lim D_{i}$ where $D_{i} \subset \Sigma_{i}^{+}$, for sufficiently large $i, \Sigma^{\prime} \cap \Sigma_{i}^{+} \neq \emptyset$. However, $\partial_{\infty} \Sigma_{i}^{+}=\Gamma_{i}^{+}$is disjoint from $\Gamma=\partial_{\infty} \Sigma^{\prime}$. Then, by Lemma 3.1, $\Sigma^{\prime}$ must be disjoint from $\Sigma_{i}^{+}$. This is a contradiction.

Similarly, this is true for $\Sigma^{-}$, too. Moreover, let $N \subset X$ be the region between $\Sigma^{+}$and $\Sigma^{-}$, i.e. $\partial N=\Sigma^{+} \cup \Sigma^{-}$. Then by construction, $N$ is also a canonical region for $\Gamma$, and for any least area plane $\Sigma^{\prime}$ with $\partial_{\infty} \Sigma^{\prime}=\Gamma, \Sigma^{\prime}$ is contained in the region $N$, i.e. $\Sigma^{\prime} \subset N$. This shows that if $\Sigma^{+}=\Sigma^{-}$, there exists a unique least area plane asymptotic to $\Gamma$. If $\Sigma^{+} \neq \Sigma^{-}$, then they must be disjoint.

Now, we are going to prove the main theorem of the paper. This theorem says that for a generic simple closed curve in the asymptotic sphere of a Gromov hyperbolic 3 -space, there exists a unique least area plane.

Theorem 3.3. Let $X$ be a Gromov hyperbolic 3-space with cocompact metric, and let $A$ be the space of simple closed curves in $S_{\infty}^{2}(X)$. Let $A^{\prime} \subset A$ be the subspace 
consisting of the simple closed curves in $S_{\infty}^{2}(X)$ bounding a unique least area plane in $X$. Then $A^{\prime}$ is generic in $A$; i.e. $A-A^{\prime}$ is a set of first category.

Proof. We will prove this theorem in 2 steps.

Claim 1. $A^{\prime}$ is dense in $A$ as a subspace of $C^{0}\left(S^{1}, S_{\infty}^{2}(X)\right)$ with the supremum metric.

Proof. Let $A$ be the space of Jordan curves in $S_{\infty}^{2}(X)$. Then we can parametrize $A$ such that $A=\left\{\alpha \in C^{0}\left(S^{1}, S^{2}\right) \mid \alpha\right.$ is an embedding $\}$.

Now, let $\Gamma_{0} \in A$ be a simple closed curve in $S_{\infty}^{2}(X)$. Since $\Gamma_{0}$ is simple, there exists a small neighborhood $N\left(\Gamma_{0}\right)$ of $\Gamma_{0}$ which is an annulus in $S_{\infty}^{2}(X)$. Let $\Gamma:(-\epsilon, \epsilon) \rightarrow A$ be a small path in $A$ through $\Gamma_{0}$ such that $\Gamma(t)=\Gamma_{t}$ and $\left\{\Gamma_{t}\right\}$ foliates $N(\Gamma)$ with simple closed curves $\Gamma_{t}$. In other words, $\left\{\Gamma_{t}\right\}$ are pairwise disjoint simple closed curves and $N\left(\Gamma_{0}\right)=\bigcup_{t \in(-\epsilon, \epsilon)} \Gamma_{t}$.

Now, $N\left(\Gamma_{0}\right)$ separates $S_{\infty}^{2}(X)$ into two parts, say $D^{+}$and $D^{-}$, i.e. $S_{\infty}^{2}(X)=$ $N\left(\Gamma_{0}\right) \cup D^{+} \cup D^{-}$. Let $p^{+}$be a point in $D^{+}$and let $p^{-}$be a point in $D^{-}$such that for a small $\delta, B_{\delta}\left(p^{ \pm}\right)$are in the interior of $D^{ \pm}$. Let $\beta$ be a proper line in $X$ asymptotic to $p^{+}$and $p^{-}$.

By Lemma 3.2, for any $\Gamma_{t}$ either there exists a unique least area plane $\Sigma_{t}$ in $X$ or there is a canonical region $N_{t}$ in $X$ between the canonical least area planes $\Sigma_{t}^{+}$ and $\Sigma_{t}^{-}$. With abuse of notation, if $\Gamma_{t}$ bounds a unique least area plane $\Sigma_{t}$ in $X$, define $N_{t}=\Sigma_{t}$ as a degenerate canonical region for $\Gamma_{t}$. Then, let $\widehat{N}=\left\{N_{t}\right\}$ be the collection of these degenerate and nondegenerate canonical regions for $t \in(-\epsilon, \epsilon)$. Clearly, a degenerate region $N_{t}$ means that $\Gamma_{t}$ bounds a unique least area plane, and a nondegenerate region $N_{s}$ means that $\Gamma_{s}$ bounds more than one least area plane. Note that by Lemma 3.1, all canonical regions in the collection are pairwise disjoint. On the other hand, by construction the proper line $\beta$ intersects all the canonical regions in the collection $\widehat{N}$.

We claim that the part of $\beta$ which intersects $\widehat{N}$ is a finite line segment. Let $P^{+}$ be the least area plane asymptotic to the round circle $\partial B_{\delta}\left(p^{+}\right)$in $D^{+}$. Similarly, define $P^{-}$. By Lemma 3.1, $P^{ \pm}$are disjoint from the collection of canonical regions $\widehat{N}$. Let $\beta \cap P^{ \pm}=\left\{q^{ \pm}\right\}$. Then the part of $\beta$ which intersects $\widehat{N}$ is the line segment $l \subset \beta$ with endpoints $q^{+}$and $q^{-}$. Let $C$ be the length of this line segment $l$.

Now, for each $t \in(-\epsilon, \epsilon)$, we will assign a real number $s_{t} \geq 0$. If there exists a unique least area plane $\Sigma_{t}$ in $X$ for $\Gamma_{t}\left(N_{t}\right.$ is degenerate), then let $s_{t}$ be 0 . If not, let $I_{t}=\beta \cap N_{t}$ and let $s_{t}$ be the length of $I_{t}$. Clearly if $\Gamma_{t}$ bounds more than one least area plane $\left(N_{t}\right.$ is nondegenerate), then $s_{t}>0$. Also, it is clear that for any $t, I_{t} \subset l$ and $I_{t} \cap I_{s}=\emptyset$ for any $t \neq s$. Then, $\sum_{t \in(-\epsilon, \epsilon)} s_{t}<C$, where $C$ is the length of $l$. This means for only countably many $t \in(-\epsilon, \epsilon), s_{t}>0$. So, there are only countably many nondegenerate $N_{t}$ for $t \in(-\epsilon, \epsilon)$. Hence, for all other $t, N_{t}$ is degenerate. This means there exist uncountably many $t \in(-\epsilon, \epsilon)$ such that $\Gamma_{t}$ bounds a unique least area plane. Since $\Gamma_{0}$ is arbitrary, this proves $A^{\prime}$ is dense in A.

Claim 2. $A^{\prime}$ is generic in $A$; i.e. $A-A^{\prime}$ is a set of first category.

Proof. We will prove that $A^{\prime}$ is a countable intersection of open dense subsets of a complete metric space. Then the result will follow by Baire category theorem. 
Since the space of continuous maps from circle to sphere $C^{0}\left(S^{1}, S^{2}\right)$ is complete with supremum metric, then the closure of $A$ in $C^{0}\left(S^{1}, S^{2}\right), \bar{A} \subset C^{0}\left(S^{1}, S^{2}\right)$ is also complete.

Now, we will define a sequence of open dense subsets $U^{i} \subset A$ such that their intersection will give us $A^{\prime}$. Let $\Gamma \in A$ be a simple closed curve in $S_{\infty}^{2}(X)$. Let $N(\Gamma) \subset S_{\infty}^{2}(X)$ be a neighborhood of $\Gamma$ in $S_{\infty}^{2}(X)$, which is an open annulus. Then, define an open neighborhood $U_{\Gamma}$ of $\Gamma$ in $A$, such that $U_{\Gamma}=\left\{\alpha \in A \mid \alpha\left(S^{1}\right) \subset\right.$ $N(\Gamma), \alpha$ is homotopic to $\Gamma$ in $N(\Gamma)\}$. Clearly, $A=\bigcup_{\Gamma \in A} U_{\Gamma}$. Now, define a proper line $\beta_{\Gamma}$ as in Claim 1, which intersects all the least area planes asymptotic to curves in $U_{\Gamma}$.

Now, for any $\alpha \in U_{\Gamma}$, by Lemma 3.2, there exists a canonical region $N_{\alpha}$ in $X$ (which can be degenerate if $\alpha$ bounds a unique least area plane). Let $I_{\alpha, \Gamma}=N_{\alpha} \cap \beta_{\Gamma}$. Then let $s_{\alpha, \Gamma}$ be the length of $I_{\alpha, \Gamma}\left(s_{\alpha, \Gamma}\right.$ is 0 if $N_{\alpha}$ is degenerate). Hence, for every element $\alpha$ in $U_{\Gamma}$, we assign a real number $s_{\alpha, \Gamma} \geq 0$.

Now, we define the sequence of open dense subsets in $U_{\Gamma}$. Let $U_{\Gamma}^{i}=\{\alpha \in$ $\left.U_{\Gamma} \mid s_{\alpha, \Gamma}<1 / i\right\}$. We claim that $U_{\Gamma}^{i}$ is an open subset of $U_{\Gamma}$ and $A$. Let $\alpha \in U_{\Gamma}^{i}$, and let $s_{\alpha, \Gamma}=\lambda<1 / i$. So, the interval $I_{\alpha, \Gamma} \subset \beta_{\Gamma}$ has length $\lambda$. Let $I^{\prime} \subset \beta_{\Gamma}$ be an interval containing $I_{\alpha, \Gamma}$ in its interior and which has length less than $1 / i$. By the proof of Claim 1, we can find two simple closed curves $\alpha^{+}, \alpha^{-} \in U_{\Gamma}$ with the following properties:

- $\alpha^{ \pm}$are disjoint from $\alpha$,

- $\alpha^{ \pm}$are lying in opposite sides of $\alpha$ in $S_{\infty}^{2}(X)$,

- $\alpha^{ \pm}$bounds unique least area planes $\Sigma_{\alpha^{ \pm}}$,

- $\Sigma_{\alpha^{ \pm}} \cap \beta_{\Gamma} \subset I^{\prime}$.

The existence of such curves is clear from the proof Claim 1, for if one takes any foliation $\left\{\alpha_{t}\right\}$ of a small neighborhood of $\alpha$ in $S_{\infty}^{2}(X)$, there are only countably many curves in the family bounding more than one least area plane, and one can choose a sufficiently close pair of curves to $\alpha$ in order to ensure the conditions above.

After finding $\alpha^{ \pm}$, consider the open annulus $F_{\alpha}$ in $S_{\infty}^{2}(X)$ bounded by $\alpha^{+}$and $\alpha^{-}$. Let $V_{\alpha}=\left\{\gamma \in U_{\Gamma} \mid \gamma\left(S^{1}\right) \subset F_{\alpha}, \gamma\right.$ is homotopic to $\left.\alpha\right\}$. Clearly, $V_{\alpha}$ is an open subset of $U_{\Gamma}$. If we can show $V_{\alpha} \subset U_{\Gamma}^{i}$, then this proves $U_{\Gamma}^{i}$ is open for any $i$ and any $\Gamma \in A$.

Let $\gamma \in V_{\alpha}$ be any curve, and let $N_{\gamma}$ be its canonical region given by Lemma 3.2. Since $\gamma\left(S^{1}\right) \subset F_{\alpha}, \alpha^{+}$and $\alpha^{-}$lie on opposite sides of $\gamma$ in $S_{\infty}^{2}(X)$. This means $\Sigma_{\alpha^{+}}$and $\Sigma_{\alpha^{-}}$lie on opposite sides of $N_{\gamma}$. By choice of $\alpha^{ \pm}$, this implies $N_{\gamma} \cap \beta_{\Gamma}=$ $I_{\gamma, \Gamma} \subset I^{\prime}$. So, the length $s_{\gamma, \Gamma}$ is less than $1 / i$. This implies $\gamma \in U_{\Gamma}^{i}$, and so $V_{\alpha} \subset U_{\Gamma}^{i}$. Hence, $U_{\Gamma}^{i}$ is open in $U_{\Gamma}$ and $A$.

Now, we can define the sequence of open dense subsets. Let $U^{i}=\bigcup_{\Gamma \in A} U_{\Gamma}^{i}$ be an open subset of $A$. Since the elements in $A^{\prime}$ represent the curves bounding a unique area minimizing plane, for any $\alpha \in A^{\prime}$, and for any $\Gamma \in A$ with $\alpha \subset N(\Gamma)$, $s_{\alpha, \Gamma}=0$. This means $A^{\prime} \subset U^{i}$ for any $i$, and hence $A^{\prime} \subset \bigcap_{i>0} U^{i}$. By Claim $1, U^{i}$ is open dense in $A$ for any $i>0$.

Now, we claim that $A^{\prime} \supset \bigcap_{i>0} U^{i}$. Assume on the contrary that there is a simple closed curve $\alpha \in \bigcap_{i>0} U^{i}$ such that $\alpha \notin A^{\prime}$. In order to prove this claim, we will construct a lower bound $\frac{1}{i_{0}}$ for $s_{\alpha, \Gamma}$ independent of $\Gamma$. In other words, we will show that for such an $\alpha$, there is an $i_{0}$ such that $s_{\alpha, \Gamma}>\frac{1}{i_{0}}$ for any $\Gamma \in A$. Hence, this shows that $\alpha \notin U^{i_{0}}$, and we will get a contradiction. 
Now, to prove this claim we give a few restrictions for the choice of $\beta_{\Gamma}$ and $N(\Gamma)$ which do not affect the arguments in the remainder of the proof. First, let $N(\Gamma)$ be $N_{\frac{\epsilon_{\Gamma}}{3}}$, where $\epsilon_{\Gamma}=\sup \left\{\epsilon \leq \epsilon_{0} \mid N_{\epsilon}\right.$ is an embedded annulus in $\left.S_{\infty}^{2}(X)\right\}$ for some fixed $\epsilon_{0}$. Hence, we have some control on the size of $N(\Gamma)$. Next, we give a restriction for the choice of $\beta_{\Gamma}$. Recall the definition of $\beta_{\Gamma}$. We modify this definition as follows: $N(\Gamma)$ separates $S_{\infty}^{2}(X)$ into two parts, say $D_{\Gamma}^{+}$and $D_{\Gamma}^{-}$, i.e. $S_{\infty}^{2}(X)=$ $N(\Gamma) \cup D_{\Gamma}^{+} \cup D_{\Gamma}^{-}$. Let $\delta_{\Gamma}^{ \pm}=\sup \left\{\delta<\delta_{0} \mid B_{\delta}\left(p_{\delta}^{ \pm}\right)\right.$is an embedded disk in $\left.D_{\Gamma}^{ \pm}\right\}$. Then let $\Delta_{\Gamma}^{ \pm}=B_{\frac{\delta_{\Gamma}^{ \pm}}{3}}\left(p_{\delta}^{ \pm}\right)$. By $[\mathrm{BM}$, we know that $X$ is homeomorphic to a unit open ball $B_{1}(0)$ in $\mathbf{R}^{3}$, and it has a natural compactification $\bar{X}$ where $\bar{X}=X \cup \partial_{\infty} X$; i.e. $\varphi: \bar{X} \rightarrow \overline{B_{1}(0)}$ is a homeomorphism. Now, we use this identification to define a subset $\mathcal{T}_{\Gamma}$ of $X$. Define $T_{\Gamma}$ in $B_{1}(0)$ as the convex hull of $\varphi\left(\Delta_{\Gamma}^{+}\right) \cup \varphi\left(\Delta_{\Gamma}^{-}\right)$in the Euclidean metric. Then let $\mathcal{T}_{\Gamma}=\varphi^{-1}\left(T_{\Gamma}\right)$. Note that $\mathcal{T}_{\Gamma}$ links $\Gamma$ (hence $\alpha$ ), and any disk bounding $\Gamma$ (hence $\alpha$ ) must intersect $\mathcal{T}_{\Gamma}$. Now, define $\beta_{\Gamma}$ as a proper line in $\mathcal{T}_{\Gamma} \subset X$ asymptotic to $p_{\Gamma}^{+}$and $p_{\Gamma}^{-}$. It is clear that the modifications of definitions of $\beta_{\Gamma}$ and $N(\Gamma)$ do not affect the remainder of the proof. With these modifications of $\beta_{\Gamma}$ and $N(\Gamma)$, we can construct the desired lower bound for $s_{\alpha, \Gamma}$ as follows. If $\alpha \notin A^{\prime}$, then by Lemma 3.2, there is a canonical region $N_{\alpha}$. Recall that $s_{\alpha, \Gamma}$ is the length of $I_{\alpha, \Gamma}=\beta_{\Gamma} \cap N_{\alpha}$. Now, we know that $\alpha \subset N_{\Gamma}$ and $\beta_{\Gamma} \subset \mathcal{T}_{\Gamma}$, and we can say that $\beta_{\Gamma} \cap \Sigma_{\alpha}^{ \pm}$must belong to compact subdisks $\Omega^{+}$in $\Sigma^{+}$and $\Omega^{-}$in $\Sigma^{-}$ independent of $\Gamma$, i.e. $\Omega^{ \pm}=\bigcup_{\alpha \in N(\Gamma)} \mathcal{T}_{\Gamma} \cap \Sigma^{ \pm}$. Then, since $\Omega^{+}$and $\Omega^{-}$are compact, $d\left(\Omega^{+}, \Omega^{-}\right)>\frac{1}{i_{0}}$ for some $i_{0}>0$. Hence, $s_{\alpha, \Gamma}>\frac{1}{i_{0}}$ for any $\Gamma$ with $\alpha \subset N(\Gamma)$. This implies that $\alpha \notin U^{i_{0}}$, and the proof follows.

As we mentioned at the beginning of the proof, since the space of continuous maps from circle to sphere $C^{0}\left(S^{1}, S^{2}\right)$ is complete with supremum metric, then the closure $\bar{A}$ of $A$ in $C^{0}\left(S^{1}, S^{2}\right)$ is also a complete metric space. Since $A^{\prime}$ is dense in $A$, it is also dense in $\bar{A}$. As $A$ is open in $C^{0}\left(S^{1}, S^{2}\right)$, this implies $U^{i}$ is a sequence of open dense subsets of $\bar{A}$. On the other hand, since $s_{\alpha, \Gamma}=0$ for any $\alpha \in A^{\prime}$, and for any $\Gamma \in A, A^{\prime}=\bigcap_{i>0} U^{i}$. Then, $A-A^{\prime}$ is a set of first category, by the Baire category theorem. Hence, $A^{\prime}$ is generic in $A$.

Hence, this proves that for a generic simple closed curve in the asymptotic sphere of a Gromov hyperbolic 3-space with cocompact metric, there exists a unique least area plane spanning the curve. In the next section, we will give some applications of this result.

Remark 3.1 (Properness). Note that the unique planes in the main theorem are all properly embedded by the existence results on properly embedded least area planes in Gromov hyperbolic spaces So1, So2 and [Co3. Note also that by the construction, $\mathrm{Co} 3$ implies that all the canonical planes given by Lemma 3.2 are also proper. For a general properness result in $\mathbf{H}^{3}$ see [Co5].

Remark 3.2. In order to prove the second part of the main theorem, one can also use Hausdorff topology on the space of simple closed curves in $S_{\infty}^{2}(X)$ instead of $A$ in $C^{0}\left(S^{1}, S^{2}\right)$. Then the same proof would go through and $A^{\prime}$ is the intersection of countable dense open subsets of $A$ in this setting, too. 


\section{Applications}

In this section, we will show that if a simple closed curve $\Gamma$ in $S_{\infty}^{2}(X)$ bounds a unique least area plane $\Sigma_{\Gamma}$ in $X$, where $X$ is a Gromov hyperbolic 3-space with cocompact metric, then for any other simple closed curve $\alpha$ not crossing $\Gamma$ in $S_{\infty}^{2}(X)$, any least area planes $\Sigma_{\alpha}$ are disjoint from $\Sigma_{\Gamma}$. In other words, the following Disjoint Planes Conjecture [Co1] is true if one of the curves bounds a unique least area plane.

Disjoint Planes Conjecture. Let $\Gamma_{1}, \Gamma_{2}$ be simple closed curves in $S_{\infty}^{2}(X)$, where $X$ is a Gromov hyperbolic 3-space with cocompact metric. If $\Gamma_{1}$ and $\Gamma_{2}$ do not cross each other (i.e. they are the boundaries of disjoint open regions in $S_{\infty}^{2}(X)$ ), then any distinct least area planes $\Sigma_{1}, \Sigma_{2}$ in $X$ with asymptotic boundary $\Gamma_{1}, \Gamma_{2}$ are disjoint.

Remark 4.1. Even though this conjecture is interesting in its own right, it has powerful topological applications. The most important application is constructing the least area representative of a 2-dimensional object in a 3-manifold, such as incompressible surfaces and genuine laminations. For example, if a closed, atoroidal 3 -manifold $M$ has a genuine lamination $\Lambda$, then by [GK, $M$ is Gromov hyperbolic. Then, if $\widetilde{\Lambda}$ induces a $\pi_{1}$-invariant family of circles in $S_{\infty}^{2}(\widetilde{M})$ (up to the continuous extension property $[\mathrm{Fe}, \mathrm{Ca}])$, then by spanning one of the curves in the family with a least area plane $\Sigma$, and considering images of $\Sigma$ under deck transformations, we get a $\pi_{1}$-invariant family of least area planes in $S_{\infty}^{2}(\widetilde{M})$. By the above conjecture, this family is pairwise disjoint; hence it can be shown that it projects down to a embedded genuine lamination with least area leaves in $M$ by using the techniques in [Co1. Similar construction works for incompressible surfaces, too, which implies a similar result of $[\mathrm{HS}$. In other words, with this conjecture, if a 2-dimensional embedded essential object $S$ in a Gromov hyperbolic manifold $M$ induces a $\pi_{1}$ invariant family of circles $\partial_{\infty} \widetilde{S}$ in $S_{\infty}^{2}(\widetilde{M})$, then it is possible to get its embedded least area representative in the 3-manifold $M$.

Now, we will prove that if one of the curves in the conjecture bounds a unique least area plane, then the conjecture is true.

Theorem 4.1. Let $\Gamma_{1}, \Gamma_{2}$ be as in the Disjoint Planes Conjecture. Let $\Gamma_{1}$ bound a unique least area plane in $X$. Then, the Disjoint Planes Conjecture is true for $\Gamma_{1}$ and $\Gamma_{2}$.

Proof. First, we will show that, for any curves $\Gamma_{1}, \Gamma_{2}$ as in the statement of the theorem, if $\Sigma_{1}^{ \pm}$and $\Sigma_{2}^{ \pm}$are the extremal least area planes with $\Sigma_{i}^{ \pm}=\Gamma_{i}$ as in Lemma 3.2, then $\Sigma_{1}^{+} \cap \Sigma_{2}^{+}=\emptyset$, and likewise $\Sigma_{1}^{-} \cap \Sigma_{2}^{-}=\emptyset$.

Let $S_{\infty}^{2}(X)-\Gamma_{i}=\Omega_{i}^{+} \cup \Omega_{i}^{-}$, and with loss of generality, further assume that $\Omega_{2}^{+} \subset \Omega_{1}^{+}$and $\Omega_{1}^{-} \subset \Omega_{2}^{-}$. (In a sense, $\Gamma_{1}$ stays below $\Gamma_{2}$ in $S_{\infty}^{2}(X)$.) By the construction in Lemma 3.2, we know that $\Sigma_{1}^{+}=\lim D_{1 i}$ for some sequence of least area disks such that $D_{1 i} \subset \Sigma_{1 i}$, where $\Sigma_{1 i}$ is a least area plane with $\partial_{\infty} \Sigma_{1 i}=\Gamma_{1 i}$ and $\Gamma_{1 i} \subset \Omega_{1}^{+}$. Similarly the same is true for $\Sigma_{2}^{+}$.

Assume that $\Sigma_{1}^{+} \cap \Sigma_{2}^{+} \neq \emptyset$. Then, by $[\mathrm{MY}]$, the intersection cannot contain a simple closed curve. Hence, the intersection must contain an infinite line $l$ with $\partial_{\infty} l \subset \Gamma_{1} \cap \Gamma_{2}$. This implies that some part of $\Sigma_{1}^{+}$must be above $\Sigma_{2}^{+}$. Recall that $\Sigma_{2}^{+}=\lim D_{2 i}$, where $D_{2 i} \subset \Sigma_{2 i}$. Moreover, $\partial_{\infty} \Sigma_{2 i}=\Gamma_{2 i}$ and $\Gamma_{2 i} \subset \Omega_{2}^{+} \subset \Omega_{1}^{+}$. This means for any $i, \Gamma_{1} \cap \Gamma_{2 i}=\emptyset$. By Lemma 3.1, this implies $\Sigma_{1}^{+} \cap \Sigma_{2 i}=\emptyset$. 
However, as some part of $\Sigma_{1}^{+}$is above $\Sigma_{2}^{+}$, and $\Sigma_{2}^{+}=\lim D_{2 i}$ where $D_{2 i} \subset \Sigma_{2 i}$, this is a contradiction. Hence $\Sigma_{1}^{+} \cap \Sigma_{2}^{+}=\emptyset$. Similarly, $\Sigma_{1}^{-} \cap \Sigma_{2}^{-}=\emptyset$ is also true for any such $\Gamma_{1}, \Gamma_{2} \subset S_{\infty}^{2}(X)$.

Now, if $\Gamma_{1}$ is a simple closed curve in $S_{\infty}^{2}(X)$ bounding a unique least area plane $\Sigma_{1}$ in $X$, then for $\Gamma_{1}$ the extremal least area planes $\Sigma_{1}^{+}$and $\Sigma_{2}^{+}$must be same, i.e. $\Sigma_{1}^{+}=\Sigma_{1}^{-}=\Sigma_{1}$. Then, if $\Gamma_{2}$ is any other simple closed curve in $S_{\infty}^{2}(X)$ which does not cross $\Gamma_{1}$ in $S_{\infty}^{2}(X)$, then by the previous paragraph, $\Sigma_{1}^{+} \cap \Sigma_{2}^{+}=\emptyset$ and $\Sigma_{1}^{-} \cap \Sigma_{2}^{-}=\emptyset$. If $\Sigma_{2}^{\prime}$ is any least area plane with $\partial_{\infty} \Sigma_{2}^{\prime}=\Gamma_{2}$, then by Lemma 3.2, $\Sigma_{2}^{\prime}$ must be between $\Sigma_{2}^{+}$and $\Sigma_{2}^{-}$. Hence, as $\Sigma_{1}^{+}=\Sigma_{1}^{-}=\Sigma_{1}$ and $\Sigma_{1}^{ \pm} \cap \Sigma_{2}^{ \pm}=\emptyset$, $\Sigma_{1} \cap \Sigma_{2}^{\prime}=\emptyset$. The proof follows.

By combining the above result with Theorem 3.3, we get the following corollary.

Corollary 4.2. The Disjoint Planes Conjecture is generically true.

Now, by using the techniques in Co1, as described in Remark 4.1, for a genuine lamination $\Lambda$ in a 3 -manifold $M$, with the corollary above, it is generically possible to modify it without changing the metric of $M$ so that the leaves of the new lamination $\Lambda^{\prime}$ are minimal. Similar, construction works for incompressible surfaces in Gromov hyperbolic 3-manifolds, too. Basically, this corollary implies that if a 2-dimensional embedded essential object $S$ in a Gromov hyperbolic manifold $M$ induces a $\pi_{1}$-invariant family of circles $\partial_{\infty} \widetilde{S}$ in $S_{\infty}^{2}(\widetilde{M})$, then it is generically possible to get its embedded least area representative in the 3-manifold $M$.

4.1. An example. Before finishing this section, we would like to give an example to show a subtlety of the Disjoint Planes Conjecture. This example shows that the problem is not local, but indeed global. If one wants to prove disjointness of these area minimizing planes, one must use the global condition, where one curve lies completely in one side of the other one, in an essential way.

We will restrict ourselves to $\mathbf{H}^{3}$ and use the upper half space model, i.e. $\mathbf{H}^{3}=$ $\left\{(x, y) \in \mathbf{R}^{2} \times \mathbf{R} \mid y>0\right\}$ and $S_{\infty}^{2}\left(\mathbf{H}^{3}\right)=\mathbf{R}^{2} \times\{0\} \cup\{\infty\}$.

Let $\Gamma_{1}=\mathbf{R} \times\{0\} \subset \mathbf{R}^{2}$, the $x$-axis, and let

$$
\begin{aligned}
\Gamma_{2}^{\prime}= & \{0\} \times[1+\sqrt{2}, \infty] \cup[(0,1+\sqrt{2}),(1,0)] \cup[(-1,0),(1,0)] \\
& \cup[(-1,0),(0,-1-\sqrt{2}) \cup\{0\} \times[-1-\sqrt{2}, \infty] .
\end{aligned}
$$

Here if $a, b \in \mathbf{R}^{2}$, then $[a, b]$ represents line segment between $a$ and $b$. See Figure 1. Note that $\Gamma_{2}^{\prime}$ is symmetric around the origin. Now, define $\Gamma_{2}$ by rounding off symmetrically around the origin the corners $\{( \pm 1,0),(0, \pm(1+\sqrt{2}))\}$ of $\Gamma_{2}^{\prime}$ in a small neighborhood of the corner points.

We claim that there exist unique area minimizing planes $M_{1}$ and $M_{2}$ spanning $\Gamma_{1}$ and $\Gamma_{2}$, and the geodesic $l=(0,0) \times \mathbf{R}^{+}=M_{1} \cap M_{2}$.

(i) There exists a unique area minimizing plane spanning $\Gamma_{i}$.

Obviously, there exists a unique area minimizing plane spanning $\Gamma_{1}$, which is the geodesic plane $\mathbf{R} \times\{0\} \times \mathbf{R}^{+} \subset \mathbf{H}^{3}$, say $M_{1}$. We will show that there exists a unique area minimizing plane spanning $\Gamma_{2}$, too. To show this, we will use the fact that if $\Omega \subset \mathbf{R}^{2} \times\{0\}$ is a star shaped domain, then there exists a unique area minimizing plane $\Sigma$ such that $\partial_{\infty} \Sigma=\partial \Omega \subset S_{\infty}^{2}\left(\mathbf{H}^{3}\right)$, [HL.

We claim that with an appropriate choice of infinity point for $S_{\infty}^{2}\left(\mathbf{H}^{3}\right), \Gamma_{2}$ will be boundary of a star shaped domain $\Omega_{2}$. Let $a=(-1-\sqrt{2}, 0) \in \mathbf{R}^{2}$. If we move our infinity to $a$ for $S_{\infty}^{2}\left(\mathbf{H}^{3}\right)$, then the bounded region in the complement of $\Gamma_{2}$ 

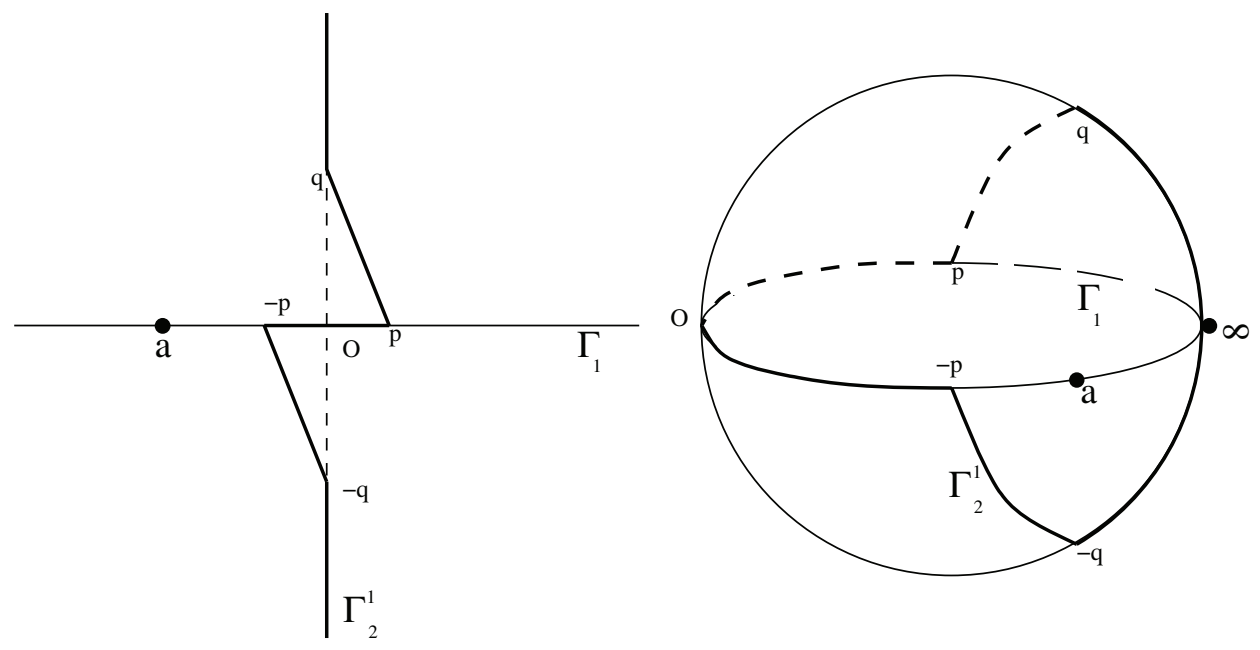

Figure $1 . \Gamma_{1}$ and $\Gamma_{2}^{\prime}$ are in $\mathbf{R}^{2} \times\{0\}$ and $S_{\infty}^{2}\left(\mathbf{H}^{3}\right)$. Here, $p=(1,0)$ and $q=(0,1+\sqrt{2})$.

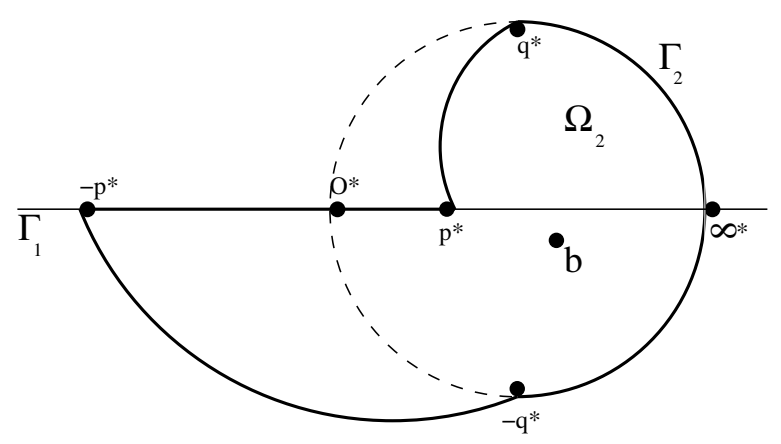

Figure 2. After suitable transformation, $\Omega_{2}$ (inside of $\Gamma_{2}$ ) is a star shaped region. Here, $x^{*}$ corresponds to point $x$ in Figure 1.

will be star shaped with an appropriate center point $b$ (see Figure 2). So, by the above fact, there exists a unique area minimizing plane with asymptotic boundary $\Gamma_{2}$, say $M_{2}$.

(ii) Symmetry.

Now, consider the isometry $\varphi$ of $\mathbf{H}^{3}$ obtained by $\pi$ rotation along the geodesic (axis of isometry) $(0,0) \times \mathbf{R}^{+}$. Note that $\varphi^{2}$ is the identity map and $\varphi\left(\Gamma_{i}\right)=\Gamma_{i}$. Since $M_{1}$ and $M_{2}$ are unique area minimizing planes spanning $\Gamma_{1}$ and $\Gamma_{2}, \varphi$ fixes $M_{1}$ and $M_{2}$, i.e. $\varphi\left(M_{i}\right)=M_{i}$.

(iii) The geodesic $l=M_{1} \cap M_{2}$. 
Claim. Let $\alpha$ and $\beta$ be two smooth curves in $S_{\infty}^{2}\left(\mathbf{H}^{3}\right)$ such that $\alpha \cap \beta=\{x, y\}$ and the intersection is transverse. If $A$ and $B$ are least area planes spanning $\alpha$ and $\beta$, then $A \cap B$ is a proper line limiting on $\{x, y\}$. If $A$ and $B$ are respectively invariant under a group $G$, then so is $A \cap B$.

Proof of the Claim. By $[\mathrm{MY}$, $A, B$ being area minimizing implies that $A \cap B$ is a locally finite union of differentiable curves and $A \cap B$ contains no simple closed curve. Since $\alpha$ is transverse to $\beta$ and $A \cap B$ are locally graphs near infinity (Theorem 2.2, [HL]), it follows that the ends of $A \cap B$ are rays limiting respectively on $x$ and $y$. These statements imply our first conclusion. The second is immediate.

Proof of (iii). Our curve $\Gamma_{2}$ is the limit of smooth curves $\beta_{i}$ such that for each $i$, $\beta_{i}$ is transverse to $\Gamma_{1}, \beta_{i} \cap \Gamma_{1}=\{0, \infty\}, \beta_{i}$ is invariant under $\pi$ rotation about the geodesic $l$ and $\beta_{i}$ is star shaped with respect to center $b$. By [A1] and [HL, $\beta_{i}$ spans a unique area minimizing plane $B_{i}$. It follows by the Claim that for each $i$, $B_{i} \cap M_{1}=l$. The standard limiting properties of area minimizing surfaces together with uniqueness of $M_{2}$ implies that $M_{2}=\lim _{i \rightarrow \infty} B_{i}$ and hence $l \subset M_{2} \cap M_{1}$. If $l \neq M_{2} \cap M_{1}$, then by [MY], $M_{2}$ is transverse to $M_{1}$ at a point missing $l$, which would imply that some $B_{i}$ had a similar property, a contradiction.

Remark 4.2. In this example, we have shown that the area minimizing planes $M_{1}$ and and $M_{2}$ intersect in the geodesic line $l$ perpendicular to $(0,0,0)$. The asymptotic curves $\Gamma_{1}$ and $\Gamma_{2}$ intersect along the union of a line segment and a point in $S_{\infty}^{2}\left(\mathbf{H}^{3}\right)$. The intersection along the line segment is not transversal, but the area minimizing planes are not disjoint. This shows that the problem is not local, but indeed global. Hence, in order to prove the Disjoint Planes Conjecture, one must use the global condition, where one curve lies completely in one side of the other one, in an essential way.

\section{Final Remarks}

In this paper, we showed that for a generic simple closed curve in the asymptotic sphere of a Gromov hyperbolic 3-space with cocompact metric there is a unique least area plane spanning the curve in the space. This is the first result in this setting, while for the analogous question in $\mathbf{H}^{3}$, there are many significant results such as $\mathrm{A} 2$, $\mathrm{HL}, \mathrm{Co} 2, \mathrm{Co} 4$. The technique which we used here is very general, and it applies to many different settings of the Plateau problem. In particular, it can naturally be generalized to the area minimizing surfaces in Gromov-Hadamard spaces which are studied by Lang in [L].

The next question is whether there exist simple closed curves in $S_{\infty}^{2}(X)$ which bound more than one least area plane in $X$, where $X$ is a Gromov hyperbolic 3 -space with cocompact metric. In the special case, when $X=\mathbf{H}^{3}$, we showed the existence of such curves in $S_{\infty}^{2}\left(\mathbf{H}^{3}\right)$ bounding more than one least area plane in $\mathbf{H}^{3}$ in [Co4]. By using the techniques in [Co4, one can easily show that if there exists a complete minimal surface $S$ with some genus in $X$ asymptotic to a simple closed curve $\gamma_{0}$ in $S_{\infty}^{2}(X)$, then there is a curve $\gamma_{t_{0}}$ in $S_{\infty}^{2}(X)$ bounding more than one least area plane in $X$, where $\left\{\gamma_{t}\right\}$ is a foliation of $S_{\infty}^{2}(X)$ with simple closed curves, where $\gamma_{s}$ is a round circle for $s \in\left[-1,-\frac{1}{2}\right]$ and $s \in\left[\frac{1}{2}, 1\right]$, i.e. $S_{\infty}^{2}(X)=\bigcup_{t=-1}^{1} \gamma_{t}\left(\gamma_{-1}, \gamma_{1}\right.$ are singular leaves). The idea is that by assuming the uniqueness for all curves, one can foliate $X$ with $\left\{\Sigma_{t}\right\}$ where $\Sigma_{t}$ is a least area plane with $\partial_{\infty} \Sigma_{t}=\Gamma_{t}$ by using Lemma 3.1, i.e. $X=\bigcup_{t=-1}^{1} \Sigma_{t}$. Now, $S$ is a minimal surface with $\partial_{\infty} S=\gamma_{0}$. 
Since $S$ has some genus, it cannot be a leaf in the foliation $\left\{\Sigma_{t}\right\}$. So, there must be a tangential intersection lying on one side with a leaf $\Sigma_{t_{1}}$. In particular, by construction there is a maximal $t_{1}$ with $t_{1} \leq \frac{1}{2}$ (or minimal $s_{1}$ with $s_{1} \geq-\frac{1}{2}$ ) for which $\Sigma_{t_{1}}$ intersects $S$. This is a contradiction by the maximum principle for minimal surfaces (Lemma 2.3).

On the other hand, in section 4, we gave an application of this generic uniqueness result by applying it to the Disjoint Planes Conjecture. As described in Remark 4.1, this conjecture has many important applications in 3-manifold topology by combining it with the techniques developed in [Co1. As Theorem 4.1 shows, to finish this conjecture the only case that needs to be ruled out is when $\Gamma_{1}, \Gamma_{2}$ are not crossing each other and they both bound more than one least area plane, i.e. $\partial_{\infty} \Sigma_{i}^{ \pm}=\Gamma_{i}$. One method to rule out this case is to "foliate" the region between $\Gamma_{1}$ and $\Gamma_{2}$ in $S_{\infty}^{2}(X)$ by simple closed curves $\Gamma_{t}$ where $1 \leq t \leq 2$, such that $\Gamma_{t} \cap \Gamma_{s}=\Gamma_{1} \cap \Gamma_{2}$ for any $1 \leq t, s \leq 2$. Hence, one gets the two families of the least area planes $\left\{\Sigma_{t}^{+}\right\}$and $\left\{\Sigma_{t}^{-}\right\}$, where $\partial_{\infty} \Sigma_{t}^{ \pm}=\Gamma_{t}$. (Otherwise, if one curve in the "foliation" $\Gamma_{t_{0}}$ bounds a unique least area plane, then this plane will be a barrier between $\Sigma_{1}^{ \pm}$and $\Sigma_{2}^{ \pm}$.) Then the idea is to analyze the intersections of these families of least area planes in order to get a contradiction by using the maximum principle for minimal surfaces.

\section{ACKNOWLEDGEMENTS}

The author is very grateful to the referee for very valuable comments and re-

marks. He would like to thank Dave Gabai for very helpful discussions during the construction of the example in section 4 .

\section{REFERENCES}

[A1] M. Anderson, Complete minimal varieties in hyperbolic space, Invent. Math. 69 (1982) 477-494. MR.679768 (84c:53005)

[A2] M. Anderson, Complete minimal hypersurfaces in hyperbolic n-manifolds, Comment. Math. Helv. 58 (1983) 264-290. MR705537 (85e:53076)

[BM] M. Bestvina, and G. Mess, The boundary of negatively curved groups, J. Amer. Math. Soc. 4 (1991) 469-481. MR1096169 (93j:20076)

[Ca] D. Calegari, Almost continuous extension for taut foliations, Math. Res. Lett. 8 (2001), no. 5-6, 637-640. MR1879807 (2002k:57070)

[Co1] B. Coskunuzer, Uniform 1-cochains and genuine laminations, Topology 45 (2006) 751784. MR2236377 (2007c:57028)

[Co2] B. Coskunuzer, Generic Uniqueness of Least Area Planes in Hyperbolic Space, Geom. \& Topology 10 (2006) 401-412. MR2224461 (2007a:53005)

[Co3] B. Coskunuzer, Properly Embedded Least Area Planes in Gromov Hyperbolic 3-Spaces, Proc. Amer. Math. Soc. 136 (2008) 1427-1432. MR2367116 (2008j:53010)

[Co4] B. Coskunuzer, On the Number of Solutions to Asymptotic Plateau Problem, eprint; math.DG/0505593

[Co5] B. Coskunuzer, Least Area Planes in Hyperbolic 3-Space are Properly Embedded, Indiana Univ. Math. J. 58 (2009) 381-392. MR 2504417 (2010b:53012)

[Co6] B. Coskunuzer, Asymptotic Plateau problem, eprint; arXiv:0907.0552

[Fe] S.R. Fenley, Foliations with good geometry, J. Amer. Math. Soc. 12 (1999), no. 3, 619-676. MR.1674739 (2000b:57041)

[Ga] D. Gabai, On the geometric and topological rigidity of hyperbolic 3-manifolds, J. Amer. Math. Soc. 10 (1997) 37-74. MR1354958 (97h:57028)

[GK] D. Gabai, W.H. Kazez, Group negative curvature for 3-manifolds with genuine laminations, Geom. Topol. 2 (1998) 65-77. MR.1619168 (99e:57023)

[Gr] M. Gromov, Hyperbolic groups, Essays in group theory, Math. Sci. Res. Inst. Publ. 8 Springer (1987) 75-263. MR919829 (89e:20070) 
[HL] R. Hardt and F.H. Lin, Regularity at infinity for absolutely area minimizing hypersurfaces in hyperbolic space, Invent. Math. 88 (1987) 217-224. MR877013 (88m:49033)

[HS] J. Hass and P. Scott, The Existence of Least Area Surfaces in 3-manifolds, Trans. AMS 310 (1988) 87-114. MR965747 (90c:53022)

[L] U. Lang, Asymptotic Plateau problem in Gromov hyperbolic manifolds, Calc. Var. Partial Differential Equations 16 (2003) 31-46. MR.1951491 (2003m:49064)

[MY] W. Meeks and S.T. Yau, The classical Plateau problem and the topology of three manifolds, Topology 21 (1982) 409-442. MR670745 (84g:53016)

[So1] T. Soma, Existence of least area planes in hyperbolic 3-space with co-compact metric, Topology 43 (2004) 705-716. MR2041639 (2005a:57017)

[So2] T. Soma, Least area planes in Gromov hyperbolic 3-spaces with co-compact metric, Geom. Dedicata 112 (2005) 123-128. MR2163893 (2006d:53007)

Department of Mathematics, Koc University, Sariyer, Istanbul 34450, Turkey

E-mail address: bcoskunuzer@ku.edu.tr 